\section{Incluindo a Temática de Drogas e Vulnerabilidade Social na Formação Profissional por meio de Ações de Extensão: o Caso da Universidade de Brasília, Campus Ceilândia}

\author{
Including Drugs and Social Vulnerability Subjects in \\ Academic Training through University Extension Project: \\ the Case of the University of Brasilia, Campus Ceilândia
}

\title{
RESUMO
}

Esse artigo tem como intuito apresentar a percepção dos participantes do projeto de extensão Roda de debate sobre crack, outras drogas e vulnerabilidades associadas, realizado na comunidade acadêmica com o objetivo de contribuir para criar, aperfeiçoar e constituir uma relação de comunicação constante e vinculada entre a universidade e a sociedade sobre o tema "drogas". Para tal, foi realizada uma pesquisa de caráter misto sequencial, de amostra aleatória, com resultados quantitativos (obtidos por questionários de auto-relato dos alunos) e qualitativos (obtidos com auxílio de entrevista semiestruturada com mediador). Os resultados demonstraram, tanto na percepção discente quanto do mediador, que esse tipo de iniciativa gera conhecimento ao estudante da universidade sobre o tema e contribui na formação acadêmica. Pode-se concluir que a atividade de extensão foi considerada um espaço para desenvolver e estimular o conhecimento, além de auxiliar na formação acadêmica dos universitários, permitindo aos estudantes a reflexão crítica sobre o tema.

Palavras-chave: Extensão Universitária. Atividade de Extensão. Formação Acadêmica. Drogas.

\section{ABSTRACT}

This article aims to present and discuss the university extension project towards the academic community, entitled Roda de debate sobre crack, outras drogas e vulnerabilidades associadas, whose goal is to create, improve and support the dialogue between

\section{Maria de Nazareth Rodrigues Malcher De O. Silva, Anne KAROLINY RODRIgUES de Paula, Vagner dos Santos, Gabriela Arantes Wagner e Andrea Donatti GALLASSI \\ Universidade de Brasília. Fa- culdade de Ceilândia, Brasília, Brasil}


university and society over several issues on drugs. To this end, data was collected among participants, using a combined methodology of quantitative (self-report questionnaires) and qualitative (semi-structured interview by a mediator) perspectives. The results indicate that both students and mediators found that such activity increase general knowledge over drug issues among students, as well as contribute to academic training. In conclusion, this university extension activity was considered as a successful alternative to develop and stimulate knowledge and critical reflection.

Keywords: University Extension. Academic Training. Drugs.

\section{INTRODUÇÃO}

\section{No início do século XIX surgiram as primeiras Escolas de Ensino Superior no} Brasil. Tal fato se deu devido a chegada da família real portuguesa ao Brasil e da necessidade de profissionais qualificados, principalmente nas áreas de Medicina, Engenharia e Direito. Posteriormente, entre a Revolução Industrial de 1930 e o Golpe Militar de 1964, surgiram várias universidades públicas no Brasil, que, a princípio, eram orientadas a dar mais ênfase ao ensino do que à pesquisa, e eram instituições isoladas, voltadas para a minoria da população $[16,20]$.

No entanto, com o movimento da reforma universitária de 1968, fixaram-se algumas normas de organização e funcionamento do ensino superior. A reforma universitária teve como base a relação entre ensino, pesquisa e extensão. Assim, a universidade deixou de ser apenas uma mera instituição de ensino, passando a ser um centro de saber [10]. Neste sentido, a formação acadêmica passou a ser humanista, crítica e reflexiva, estimulando a vivência de distintas formas de pensar, produzir e socializar o saber.

Seguindo a reforma universitária, o Projeto Político-Pedagógico Institucional (PPPI) da Universidade de Brasília (UnB) tem como princípio a indissociabilidade entre ensino, pesquisa e extensão. Além disso, atua em consonância com os pilares de base para a constituição e avanços do ensino superior brasileiro, ou seja, o ensino, a pesquisa e a extensão, que sustentam a formação acadêmica [5]. No PPI entende-se como extensão (1) o processo de ensino e aprendizagem associados à pesquisa e ações junto à comunidade, (2) a interdisciplinaridade e a flexibilidade como procedimentos contemporâneos de construção do saber, (3) a contextualização social e histórica da ciência e (4) a diversidade como proposta de ação e inserção.

Neste artigo descreveremos uma das ações de extensão desenvolvidas pela Faculdade Ceilândia (FCE), um campus descentralizado da UnB que foi planejado para ampliar a oferta de ensino superior à população residente no Distrito Federal e municípios da Região Integrada de Desenvolvimento do Entorno do Distrito Federal (RIDE-DF). Todos os cursos oferecidos pela FCE são voltados para a área de saúde (Terapia Ocupacional, Fisioterapia, Saúde Coletiva, Enfermagem, Farmácia e Fonoaudiologia), com enfoque interdisciplinar, reforçando a capacidade técnica e a necessidade de articulação e diálogo constante com as redes do Sistema Único de Saúde (SUS) e do Sistema Único de Assistência Social (SUAS), principalmente em relação aos temas voltados à vulnerabilidade social, os quais incluem a questão do uso de 
álcool e outras drogas e suas relações. Tal medida se justifica tendo em vista que tais temas representam grandes desafios sociais e de saúde pública desta região, além de serem anteriormente negligenciados nos currículos acadêmicos [4, 11].

\section{Centro Regional de Referência sobre Drogas e Vulnerabilidades Associadas - Faculdade de Ceilândia (CRR-UnB-FCE)}

Estabelecido em 2012 por meio de uma parceria entre a Secretaria Nacional de Políticas sobre Drogas (SENAD), do Ministério da Justiça (MJ), e a Universidade de Brasília, o CRR-UnB-FCE se configura como um programa de extensão que surgiu com o objetivo de oferecer formação permanente aos profissionais que atuam nas redes do SUS, SUAS, Ministério Público, Segurança Pública e Poder Judiciário, qualificando e potencializando a abordagem junto aos usuários de álcool e outras drogas e seus familiares. A região abrangida pelo CRR-UnB-FCE compreende três municípios da RIDE-DF localizados no estado de Goiás - Águas Lindas, Valparaíso e Luziânia - e uma Região Administrativa localizada no Distrito Federal - Brazlândia. Esta região do entorno de Brasília se caracteriza pela alta vulnerabilidade típica de regiões de fronteira no que se refere à violência, muitas vezes associada ao tráfico de drogas, e à carência no acesso a bens sociais, culturais, de saúde e educação.

No CRR-UnB-FCE a formação/capacitação profissional acontece por meio de cursos temáticos com duração de 60 horas, sendo 30 horas presenciais no modelo de semana intensiva (crash-course) e as demais 30 horas dedicadas ao desenvolvimento das atividades de matriciamento in loco [11]. As atividades de matriciamento aqui referem-se ao suporte técnico e orientação clínica às equipes participantes dos cursos para o acompanhamento e tratamento de usuários de drogas e seus familiares, assim como para a implementação de ações de promoção de saúde mental. O matriciamento, dessa forma, visa proporcionar apoio e autonomia aos profissionais das diferentes equipes participantes das capacitações nas ações sobre álcool, crack e outras drogas por meio de estratégias como supervisão, discussões e estudos de caso clínico, possibilitando, assim, o aparato e suporte para formação continuada, complementando e articulando os conteúdos aprendidos na fase presencial.

O CRR-UnB-FCE conta com uma equipe de sete professores, dois consultores científicos, dois profissionais da área de saúde, um profissional administrativo e 10 alunos que desenvolvem pesquisas acadêmicas em diferentes níveis (pós-doutorado, doutorado, mestrado e iniciação científica), além dos projetos de extensão em diferentes contextos a ele vinculados. Estes projetos visam, especificamente, (1) ao mapeamento da rede integral de assistência ao usuário de álcool, crack e outras drogas e seus familiares na região de atuação do CRR-UnB-FCE; (2) à inclusão de usuários de álcool e outras drogas em projetos de geração de renda e (3) à Roda de Debate: álcool, crack, outras drogas e vulnerabilidades associadas, objeto desse artigo.

Sendo assim, o projeto de extensão Roda de Debate: álcool, crack, outras drogas e vulnerabilidades associadas, realizado na comunidade acadêmica do campus da UnB-FCE, será apresentado e discutido. O objetivo deste projeto é o de contribuir para criar, aperfeiçoar e constituir uma relação de comunicação constante e vinculada entre 
a universidade e a sociedade sobre o tema "drogas" na forma de debates, nos quais a comunidade acadêmica e o mediador exploram os limites práticos e teóricos sobre diferentes aspectos que permeiam o tema, permitindo um espaço relevante para a formação acadêmica.

\section{MÉTODOS}

\section{Roda de Debate: Álcool, Crack, Outras Drogas e Vulnerabilidades Associadas}

As atividades apresentadas neste manuscrito foram desenvolvidas em 18 encontros (com duas horas de duração cada um) entre os meses de maio e outubro de 2013, no campus da UnB-FCE. Nesses encontros foram realizados diferentes debates a partir do tema drogas, conforme descritos a seguir: (1) políticas públicas qualificadas e estrategicamente eficazes; (2) contextualização do universo da juventude e a complexidade do uso de droga; (3) atuação dos profissionais da saúde em grupos vulneráveis; (4) trabalho interdisciplinar dos Centros de Atenção Psicossocial Álcool e Drogas (CAPS ad); (5) o meio sociocultural como influência na formação de conceitos sobre usuários de drogas; (6) Alcoólicos Anônimos (AA) e Narcótico Anônimos (NA) como recurso de ajuda para pessoas com problemas relacionados ao uso de álcool e outras drogas; (7) os diferentes processos clínicos da toxicomania e possibilidades de intervenção; (8) a singularidade no cuidado do usuário com direitos e escolhas; (9) vivência das pessoas em situação de rua e as possibilidades para o cuidado.

\section{Aspectos Éticos}

Esse estudo teve aprovação do Conselho de Ética e Pesquisa (CEP) do Programa de Extensão do CRR-FCE-UnB sob protocolo no 353.571 de 25 de julho de 2013.

\section{Participantes}

135 foi o total de participantes dos encontros; sendo cinco membros da equipe do projeto, sete mediadores convidados (profissionais das redes SUS, SUAS, membros do AA/NA e professores universitários) e 123 alunos de graduação de diferentes cursos e instituições. Cada encontro contou com uma média de 30 participantes, sendo a grande maioria estudantes universitários.

\section{Coleta de Dados}

A coleta dos dados ocorreu com uma amostra aleatória de 10 estudantes participantes da Roda e de três mediadores, por meio de questionários e entrevistas semiestruturadas com os estudantes e mediadores, respectivamente. O questionário apresentou sete questões fechadas de múltipla escolha e a entrevista contou com perguntas abertas, buscando caracterizar a percepção sobre a participação na Roda de Debate, assim 
como sobre os benefícios e dificuldades da atividade de extensão sobre os temas "drogas" e "vulnerabilidade social". A aplicação dos instrumentos foi feita por estudantes bolsistas supervisionados pelos coordenadores do projeto e do programa de extensão.

\section{Análise dos Dados}

Os dados quantitativos foram digitados e tabulados pelo programa Excel $2011^{\circledR}$ e descritos por médias, desvio e percentuais. A análise dos dados qualitativos seguiu cinco estágios, usando a seguinte estrutura: Familiarização, Identificação, Indexação, Mapeamento e Interpretação. Essa estrutura compreende desde a imersão dos dados brutos, identificação de todos os tópicos, conceitos e temas-chaves pelos quais os dados possam ser examinados e referenciados, aplicação sistemática da estrutura ou índice temático, rearranjo dos dados, definição e mapeio dos fenômenos e suas associações [17].

\section{RESULTADOS E DISCUSSÃO}

A participação dos estudantes da CRR-UnB-FCE dos seis diferentes cursos ficou assim distribuída: 1\% do curso de Farmácia; 9\% de Enfermagem; 22\% de Saúde Coletiva; 24\% de Fisioterapia; e 44\% de Terapia Ocupacional (TO).

Embora a missão do campus Ceilândia seja a formação com vistas ao SUS e a interlocução comunitária, observou-se no estudo que um número pequeno de estudantes do curso de Farmácia participou dos encontros da Roda de Debate, sendo a maioria do curso de TO. Por outro lado, isso pode indicar a grande ênfase em aspectos psicossociais oferecidos na formação no contexto nacional entre as profissões focadas no cuidado de pessoas, como é o caso da Terapia Ocupacional $[1,2,8]$.

A pouca participação de alunos do curso de Farmácia pode indicar, também, uma pouca interlocução e debate entre os aspectos psicossociais e a abordagem biológica do uso de drogas. Nesse sentido, é necessário preparar os estudantes em uma formação mais ampla e abrangente que proporcione o encontro de diferentes áreas do conhecimento, combinando saberes que permitam entender esse fenômeno sob diferentes perspectivas $[4,11]$.

Houve uma limitação no que se refere à participação de estudantes de outras Instituições de Ensino Superior (IES). Em outra atividade sobre drogas semelhantes a esta e também promovida pelo CRR-UnB-FCE, foi identificado como aspecto positivo o encontro de estudantes de diferentes áreas e IES, uma vez que o conhecimento era compreendido e debatido a partir de diferentes perspectivas [18]. Os participantes também indicaram a possibilidade de que os encontros contassem com mais participantes discentes e representantes da rede e da comunidade local. Dessa forma, todos estariam, ao mesmo tempo, conhecendo os projetos da Universidade e compartilhando a realidade e desafios sociais da rede de serviços.

Com relação às características dos universitários participantes, observou-se que há quase uma distribuição homogênea entre os estudantes do terceiro (16\%), quarto 
(17\%), quinto (19\%), sexto (19\%) e sétimo (16\%) períodos. Observa-se também que os discentes do primeiro (2\%) e segundo (7\%) períodos tiveram um percentual baixo de participação na Roda de Debate. Esse acontecimento pode estar relacionado ao processo de adaptação ao cotidiano e a descoberta da universidade.

Como indicado em outro estudo [3], em relação à opinião dos estudantes, observou-se uma predominância daquelas que consideraram como interessantes os temas debatidos na Roda de Debate. Este dado é confirmado pelo alto índice de retorno dos mesmos estudantes ( $80 \%$ ) à Roda de Debate, com participação de três ou mais vezes. Em relação ao papel da Roda de Debate para os estudantes, temos que a participação se pauta nas seguintes categorias: estimular a crítica (5\%); tirar dúvida (10\%); curiosidade $(10 \%)$; promover a reflexão $(20 \%)$; complementar a formação $(63 \%)$; reflexão sobre a prática profissional (34\%); para aumento de conhecimentos específicos (37\%).

A partir destes dados, observou-se que essa atividade é relevante e auxilia no processo de formação com uma participação ativa. Isso pode estar associado ao fato de que a Roda de Debate facilitou a realização de uma conversa aberta entre mediadores e estudantes sobre conteúdos diversos, como um espaço dinâmico e distinto do espaço e estrutura das disciplinas desenvolvidas no contexto formal de ensino em sala de aula. Este processo é confirmado pelo Mediador em relato a seguir, quando ele comenta sobre os projetos de extensão, como a Roda de Debate, e sua contribuição para a formação:

"Auxilia... (pausa) auxilia, por que o conhecimento não pode ser monotemático, ele não está só na sala de aula, ele está muitas vezes em algo que não cabe na sala de aula, que é essa troca menos diretiva, né... o debate tem uma troca de impressões, de leituras, e é muito mais livre. Eu acho que auxilia sim”. (MEDIADOR).

Esse aspecto das limitações do modelo clássico de educação tem sido constantemente questionado no âmbito acadêmico e representa um dos grandes desafios da universidade do futuro $[12,19,21]$. Nesse sentido, a Roda de Debate é um ambiente favorável para a discussão das dificuldades e construção de conhecimentos sobre o abuso de drogas e suas consequências, pautados na perspectiva dos direitos humanos, de saúde e cidadania, evitando a abordagem de cunho moral e repressivo que por vezes permeia esse debate. Essa temática encontra um currículo limitado nos cursos da área de saúde [4] e uma alta carga moral de concepções que influenciam desde a elaboração de políticas públicas [15] até a prática clínica [14].

Ainda, a Roda de Debate proporciona um espaço para reflexões, críticas e curiosidades por tratar de temas que, muitas vezes, geram polêmica como, por exemplo, a legalização da maconha e a internação compulsória de usuários de drogas, ocorrendo uma diversidade de opiniões, sentimentos, moralidades expressas e concepções que são compartilhadas de forma horizontal, gerando um movimento de circularidade de opiniões, ideias e conceitos sobre o tema. Nesse sentido, complementa de forma ampla e qualificada a formação acadêmica, pois possibilita modificar as práticas pedagógicas atuais:

(...) A formação profissional e qualquer tipo de formação. Eu acho que têm até pouco, eu acho que... (pausa) os temas e a própria roda de debate se elas fossem mais frequentes, se tivessem outras rodas de debates eu acho que a formação dos alunos seria outra (MEDIADOR). 
A partir da referência do Mediador sobre este aspecto, pode-se perceber a relevância desse espaço e, adicionalmente, a necessidade de projetos de extensão voltados para o cotidiano universitário e a relação necessária com a rede intersetorial de serviços.

O fato da maioria dos estudantes considerar que a Roda de Debate contribui para a formação acadêmica está relacionado ao fato de que $63 \%$ deles acharam que a Roda é importante e $34 \%$ por a considerarem relevante na prática profissional. Assim, como indicado em outros estudos, $[6,7,13,18]$, um dos Mediadores reconhece que as atividades de extensão universitária têm um papel fundamental na formação, como segue:

Os projetos de extensão contribuem para a interdisciplinaridade e para a transversalidade de temas e, dessa maneira, contribui para formação acadêmica do aluno. Esse tipo de projeto a gente vê um resultado (MEDIADOR).

A participação dos estudantes em projetos de extensão pode significar um ponto de partida para uma modificação na sua formação acadêmica e profissional, possibilitando-os desenvolver uma intervenção humanizada e integradora, uma vez que o aprendizado e as vivências consideram o contexto real das necessidades de saúde, sociais, culturais e educacionais das pessoas dentro de seus territórios, e não somente foca em simulações ou intervenções em ambientes controlados [18].

Pode significar, também, um espaço de socialização e troca de informações, configurando-se como um meio de se aprender de forma mais dinâmica, prazerosa e factível do que somente em salas de aula, laboratórios ou clínicas-escola.

\section{CONSIDERAÇÕES FINAIS}

Ao longo da realização dos encontros da Roda de Debate, da coleta dos dados, da análise dos resultados e da redação dos resultados, deu-se conta de que é necessário seguir, ampliar e aprimorar a experiência da extensão universitária, oferecendo um caminho aberto para a circulação da comunidade e de universitários nesta discussão.

Consideramos, finalmente, que ainda há grandes desafios para seguir abordando um tema de grande relevância social, principalmente em um contexto de grande vulnerabilidade, como o caso de Ceilândia e a região da RIDE/DF. No entanto, acreditamos que se trata de um dever, como professores universitários, cumprir com o papel político e pedagógico na formação de futuros profissionais, para que estes tenham ferramentas necessárias para abordar e intervir de maneira abrangente e consistente, considerando os diferentes aspectos constituintes das necessidades das pessoas dentro de seus territórios social, cultural e educacional. 


\section{REFERÊNCIAS}

[1] ALMEIDA, $M$ et al. Terapia Ocupacional e pessoas em situação de rua: criando oportunidades e tensionando fronteiras. Cadernos de Terapia Ocupacional UFScar. São Carlos, v.19, n.3, 2001. Disponível em: <http://www.cadernosdeterapiaocupacional.ufscar.br/index.php/cadernos/article/view/505>. Acesso em 18 set. 2014. DOI: 10.4322/cto 2011.008

[2] ARAUJO, Ket al. Experiências da Terapia Ocupacional em um núcleo de apoio à saúde da família (NASF) do Distrito Federal. Revista Eletrônica Gestão \& Saúde. Brasília, v.4, n.3, 2013. Disponível em: <http://gestaoesaude.unb.br/ index.php/gestaoesaude/article/view/539>. Acesso em 18 set. 2014.

[3] ASINELLI-LUZ, A. A extensão universitária enquanto fonte de conhecimento nos temas drogas, gênero e sexualidade. Extensão em Foco. Curitiba, v.o, n.1, 2008. Disponível em: <http://ojs.c3sl.ufpr.br/ojs2/index.php/ extensao/article/view/11766>. Acesso em 18 set. 2014. DOI: http://dx.doi. org/10.5380\%2Fef.voi1.11766

[4] BARROS, H. et al. Neuroscience Education for Health Profession Undergraduates in a Call-Center for Drug Abuse Prevention. Drug and Alcohol Dependence. v.98, n.3, 2008. Disponível em: $<$ http://www.drugandalcoholdependence.com/article/So376-8716(08)o0248-2/pdf > . Acesso em 18 set. 2014.

[5] BRASIL. Universidade de Brasília. Projeto Político Pedagógico Institucional da Universidade de Brasília. Brasília: UnB, 2011.

[6] BORGES, M. Apreendendo à cidadania: Extensão universitária e direitos humanos. Revista da Faculdade de Direito da UFPR. Curitiba, v.43, n.o, 2005. Disponível em: <http://ojs.c3sl.ufpr.br/ojs/index.php/direito/article/view/7035>. Acesso em 18 set. 2014. DOI: http:// dx.doi.org/10.5380\%2Frfdufpr.v43io.7035

[7] CASTRO, L. A universidade, a extensão universitária e a produção de conhecimentos emancipadores: ainda existem utopias realistas. s.n. 2004.

[8] COSTA, S. Terapia Ocupacional social: dilemas e possibilidades da atuação junto a Povos e Comunidades Tradicionais. Cadernos de Terapia Ocupacional da UFSCar. São Carlos, v.20, n.1, 2012. Disponível em: http://www.cadernosdeterapiaocupacional.ufscar.br/index.php/cadernos/article/view/547>. Acesso em 18 set. 2014. DOI: http://dx.doi.org/10.4322/cto.2012.005

[9] CUNHA, M. Conta-me agora!: As narrativas como alternativas pedagógicas na pesquisa e no ensino. Revista da Faculdade de Educação. São Paulo, v.23, n. 1-2, 1997. Disponível em: http://www.scielo.br/scielo.php?script=sci_arttex t\&pid=So102-25551997000100010 >. Acesso em 18 set. 2014. DOI: http://dx.doi. org/10.1590/So102-25551997000100010

[10] FRAGA, M. D.; SIANO, L. M. F. A ideia da Universidade na reforma universitária de 1968. Revista de Administração Pública. Rio de Janeiro, v. 25 , n. 3, 1991. Disponível em: < bibliotecadigital.fgv.br/ojs/index.php/rap/article/ viewArticle/8945>. Acesso em 18 set. 2014. 
[11] GALLASSI, A. DOS SANTOS, V. O. abuso de drogas: desafios e opções para a prática do profissional de saúde no Brasil. Brasília Médica. Brasília, v.50, n.1, 2013. Disponível em: <http://www.ambr.org.br/brasilia-medica-volume-50-1-2013/ > . Acesso em 18 set. 2014. DOI: 10.14242/2236-5117.2013v5on1a33p51

[12] HMELO-SILVER et al. Problem-Based Learning: What and How Do Students Learn? Educational Psychology Review. New Brunswick, v.16, n.3, 2004. Disponível em: <http:// kanagawa.lti.cs.cmu.edu/olctso9/sites/default/files/Hmelo-Silver_2004.pdf $>$. Acesso em 18 set. 2014. DOI: 1040-726X/04/0900-0235/o

[13] HENNINGTON, É. Acolhimento como prática interdisciplinar num programa de extensão universitária. Cadernos de Saúde Pública. Rio de Janeiro, v.21, n.1, 2005. Disponível em: <http://www.scielo.br/scielo.php?script=sci arttext\&pid=So102-311X2005000100028\&lng=en\&nrm=iso $>$. Acesso em 18 set. 2014. DOI: http://dx.doi.org/10.1590/So102-311X2005000100028

[14] LUOMA, J et al. Reducing Self-Stigma in Substance Abuse through Acceptance and Commitment Therapy: Model, Manual Development, and Pilot Outcomes. Addiction Research \& Theory. Reno, v.16, n.2, 2008. Disponível em: <http://informahealthcare.com/doi/abs/10.1080/16066350701850295>. Acesso em 18 set. 2014. DOI: 10.1080/16066350701850295

[15] MACGREGOR, S. Barriers to the Influence of Evidence on Policy: Are Politicians the Problem? Drugs: Education, Prevention, and Policy. London, v.20, n.3, 2013. Disponível em: <http://informahealthcare.com/doi/abs/10.3109/09687637.201 2.754403 >. Acesso em 18 set. 2014. DOI: 10.3109/09687637.2012.754403

[16] MARTINS, ACP. Ensino superior no Brasil: da descoberta aos dias atuais. Acta Cir. Bras., São Paulo, v. 17, supl. 3, 2002. Disponível em: <http://www.scielo. $\mathrm{br} /$ scielo.php?script=sci_arttext\&pid=S0102-86502002000900001\&lng=en\& $\mathrm{nrm}=\mathrm{iso} \& \mathrm{t} I n g=\mathrm{pt}>$. Acesso em 18 set. 2014. DOI: http://dx.doi.org/10.1590/ So102-86502002000900001

[17] POPE, C., MAYS, N. Pesquisa qualitativa na atenção à saúde. Porto Alegre: Artmed Editora, 2005.

[18] PORTAL, MM et al. Proposta de aprendizagem sobre Drogas de Abuso por meio de uma Liga de Dependencia Quimica. Revista ABEM. In: 44 Congresso Brasileiro de Educação Médica, 2006.

[19] SMITH, Ket al.Pedagogies of Engagement: Classroom-Based Practices. Journal of Engineering Education. v.94, n.1, 2005. Disponível em: <http://onlinelibrary.wiley.com/doi/10.1002/j.2168-9830.2005.tboo831.x/abstract>. Acesso em 18 set. 2014. DOI: 10.1002/j.2168-9830.2005.tboo831.x

[20] STALLIVIERI, L. O sistema de Ensino Superior do Brasil: características, tendências e perspectivas. Caxias do Sul, RS, 2009.

[21] WALLERSTEIN, N \& BERNSTEIN, E. Empowerment Education: Freire's Ideas Adapted to Health Education. Health Education \& Behavior. v.15, n.4, 1988. Disponível em: <http://heb.sagepub.com/content/15/4/379.refs > . Acesso em 18 set. 2014. DOI: 10.1177/109019818801500402 


\section{AGRADECIMENTOS}

Os autores agradecem ao Ministério da Justiça e à Secretaria Nacional de Políticas sobre Drogas (SENAD) pelo apoio financeiro para desenvolvimento das atividades e do manuscrito. Assim como ao Decanato de Extensão (DEX) da Universidade de Brasília pelo suporte institucional para implementação e manutenção das atividades.

MARIA DE NAZARETH RODRIGUES MALCHER DE O. SILVA professora assistente no curso de Terapia Ocupacional na Faculdade de Ceilândia da Universidade de Brasília - e-mail: malchersilva@unb.br

ANNE KAROLINY RODRIGUES DE PAULA terapeuta ocupacional pela Faculdade de Ceilândia da Universidade de Brasília

VAGNER DOS SANTOS professor assistente no curso de Terapia Ocupacional na Faculdade de Ceilândia da Universidade de Brasília. Doutorando no Phoenix Dynamics of Health and Welfare Program, Linköping University, Suécia

GABRIELA ARANTES WAGNER pós-doutoranda no Departamento de Epidemiologia da Faculdade de Saúde Pública da Universidade de São Paulo (FSP-USP)

ANDREA DONATTI GALLASSI coordenadora do Centro Regional de Referência sobre Drogase Vulnerabilidades Associadas e professora adjunta no curso de Terapia Ocupacional na Faculdade de Ceilândia da Universidade de Brasília 